\title{
Correlation between MRI results and intraoperative findings in patients with silicone breast implants
}

This article was published in the following Dove Press journal:

International Journal of Women's Health

30 July 2014

Number of times this article has been viewed

\author{
Nicole Lindenblatt ${ }^{1}$ \\ Karem El-Rabadi ${ }^{2}$ \\ Thomas H Helbich ${ }^{2}$ \\ Heinrich Czembirek ${ }^{3}$ \\ Maria Deutinger ${ }^{4}$ \\ Heike Benditte-Klepetko ${ }^{5}$ \\ 'Division of Plastic and Hand \\ Surgery, Department of Surgery, \\ University Hospital Zurich, Zurich, \\ Switzerland; ${ }^{2}$ Department of \\ Biomedical Imaging and Image-guided \\ Therapy, Division of Molecular and \\ Gender Imaging, Medical University \\ of Vienna - General Hospital Vienna, \\ ${ }^{3}$ Department of Radiology, Hospital \\ Wiener Privatklinik, ${ }^{4}$ Department of \\ Plastic and Reconstructive Surgery, \\ Hospital Rudolfstiftung, Vienna, \\ Austria; ${ }^{5}$ Department of Plastic and \\ Reconstructive Surgery, Erasmus \\ Medical Center, Rotterdam, the \\ Netherlands
}

Background: Silicone gel breast implants may silently rupture without detection. This has been the main reason for magnetic resonance imaging (MRI) of the augmented or reconstructed breast. The aim of the present study was to investigate the accuracy of MRI for implant rupture.

Methods: Fifty consecutive patients with 85 silicone gel implants were included in the study. The mean age of the patients was 51 (range 21-72) years, with a mean duration of implantation of 3.8 (range 1-28) years. All patients underwent clinical examination and breast MRI. Intraoperative implant rupture was diagnosed by the operating surgeon.

Results: Nineteen of the 50 patients suffered from clinical symptoms. An implant rupture was diagnosed by MRI in 22 of 85 implants (26\%). In seven of 17 removed implants (41\%), the intraoperative diagnosis corresponded with the positive MRI result. However, only $57 \%$ of these patients were symptomatic. Ultrasound imaging of the harvested implants showed signs of interrupted inner layers of the implant despite integrity of the outer shell. By microsurgical separation of the different layers of the implant shell, we were able to reproduce this phenomenon and to produce signs of implant rupture on MRI.

Conclusion: Our results show that rupture of only the inner layers of the implant shell with integrity of the outer shell leads to a misdiagnosis on MRI. Correlation with clinical symptoms and the specific wishes of the patient should guide the indication for implant removal.

Keywords: breast implant, rupture, silicone gel, magnetic resonance imaging, linguine, keyhole

\section{Introduction}

The total number of patients with breast implants worldwide is estimated by the US Food and Drug Administration to be 5-10 million. ${ }^{1}$ Approximately 250,000-340,000 people in the USA receive breast implants each year. ${ }^{2}$ The most frequent long-term complications of implant placement include capsular contracture and implant rupture. Rupture is defined as a disruption of the implant shell, including focal rupture to large tears, and may be the result of trauma, deterioration of the implant shell with time, or manufacturing defects. Plastic surgeons are responsible for dealing with questions about potential negative health effects in the event of undetected implant rupture. Therefore, implant integrity and silent implant ruptures are important issues. In addition, it has been questioned repeatedly whether women with ruptured implants may be at risk for immunological reactions due to the exposed free silicone which may cause systemic diseases. ${ }^{3,4}$ Several imaging methods, including mammography, ultrasonography, computed tomography, and magnetic resonance imaging (MRI), have been used to assess the integrity of breast implants. In previous studies, MRI has been shown to be superior in detection of breast implant rupture compared with other methods..$^{5-8}$ 
Because progress in detection of rupture has not been accompanied by knowledge of the frequency and severity of complications associated with rupture, discussion continues regarding the most appropriate way to deal with presumed implant ruptures detected on MRI examination in symptomatic and asymptomatic patients. The potential for unnecessary surgical intervention in the event of a false positive MRI diagnosis, ie, an examination positive for implant rupture but without correlation at the time of explantation, is a cause for concern. On the other hand, false negative MRI results, ie, failure to detect an implant rupture that is actually present, may expose the patient to the potential negative sequelae of extracapsular implant rupture and silicone migration.

Therefore, the aim of the present study was to investigate the congruence of MRI results and intraoperative findings in the diagnosis of breast implant rupture in both symptomatic and asymptomatic patients. Further, we attempted to simulate potential causes for the appearance of false implant rupture signs by experimental implant dissection.

\section{Materials and methods}

\section{Patients}

Fifty consecutive patients with 85 silicone gel-filled breast implants consulting the outpatient clinic between July 2001 and April 2003 were included in the study. Of those 50 patients, 25 had previously undergone implantation of silicone gel-filled breast prostheses for reconstructive reasons and 25 for cosmetic reasons. All patients with cosmetic breast augmentation had bilateral breast implants, while patients with breast reconstruction had unilateral implants. The mean age of the patients was 51 (range 21-72) years, with a mean implant duration of 3.8 (range 0.2-28) years. A clinical examination was performed in all patients, followed by MRI of the breasts.

\section{Clinical examination}

All patients underwent a standardized clinical breast examination by a physician. As clinical symptoms of potential implant rupture, the appearance of visible folds, shape change or volume reduction of the implant, capsular contracture and pain were defined as criteria (Figure 1A and B).

\section{MRI}

All MRI scans were performed on a 1.0 Tesla MR scanner (Gyroscan, T10-NT, Philips Healthcare, Eindhoven, the Netherlands) using a dedicated breast coil in the prone position. The imaging protocol consisted of five sequences, including a T2-weighted turbo-spin-echo sequence in the
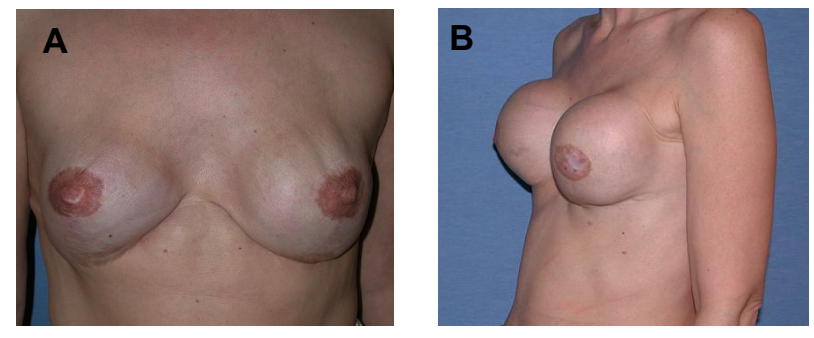

Figure I Patient presenting with clinical symptoms indicated by $(\mathbf{A})$ visible rippling and shape changes of the implant and (B) capsular contracture grade 4 .

axial and sagittal planes, a three-dimensional $\mathrm{T} 2$-weighted fast-field-echo in the sagittal plane, a T1-weighted turbo-spinecho sequence in the sagittal plane, and a three-dimensional T1-weighted fast-field-echo sequence. Both T1-weighted and T2-weighted fast-field-echo sequences used water suppression for better visualization of silicone. MRI images were read and interpreted before surgery by two independent experienced radiologists. Signs of intracapsular rupture of the implant were the keyhole and linguine signs. The keyhole sign (also known as the inverted teardrop sign) results from extraprosthetic silicone gel trapped in an invagination of the implant shell after interruption of the envelope (Figure 2A). It represents an uncollapsed rupture in which the silicone shell still covers the viscous core of the implant. On the other hand, the linguine sign is represented by multiple curvilinear low signal intensity lines seen within the high signal intensity silicone gel (Figure 2B). The curvilinear lines represent the collapsed implant shell floating within the silicone gel surrounded by the fibrous capsule. ${ }^{9}$ In contrast with this are radial folds of the implant usually seen during MRI, which are of no pathological significance (Figure 2C).

\section{Intraoperative diagnosis}

For intraoperative diagnosis, we removed the implants within their fibrous capsule to prevent iatrogenic damage of the implant shell. The implant capsule was then opened carefully. Criteria for determination of implant rupture at the time of explantation were a ruptured implant shell with silicone leakage and/or subsequent calcification of the fibrous capsule. In the event of a false positive MRI result, we additionally performed ultrasound imaging of the harvested implant.

\section{Experimental simulation of implant rupture signs}

By outer compression of a new unused implant, we produced complex radial folds in the MRI to analyze the source of a false positive keyhole sign. Additionally, we separated the different shell layers of new silicone gel-filled breast implants 

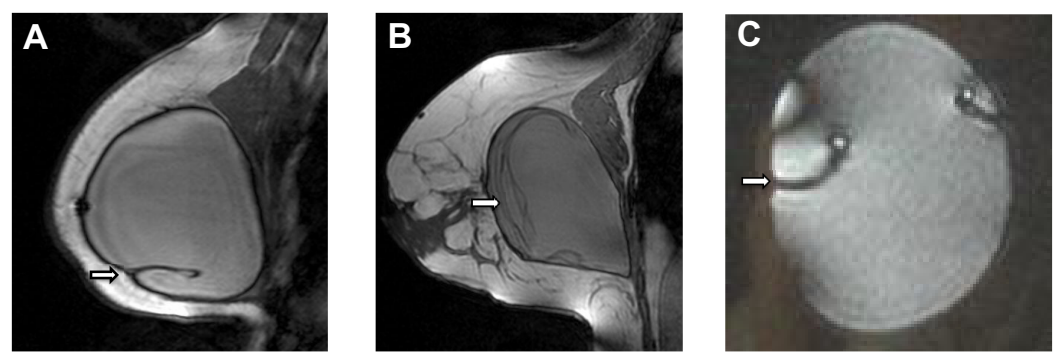

Figure 2 Magnetic resonance imaging.

Notes: (A) Magnetic resonance image of the keyhole or inverted teardrop sign indicating intracapsular free silicone trapped in a fold of the implant shell (arrow). (B) Magnetic resonance image of the linguine sign caused by a collapsed rupture of the implant shell, which then floats within the liquid silicone gel (arrow); (C) for comparison nonpathological complex radial folds of the implant on magnetic resonance imaging by outer compression of an intact silicone gel-filled breast implant (arrow).

microsurgically to analyze the potential underlying cause of a false positive linguine sign. The implants were then examined by MRI to verify the imaging changes.

\section{Results}

\section{Clinical examination}

Nineteen of the $50(38 \%)$ patients had at least one clinical symptom at the time of examination. Of these, 17 underwent surgery with the suspicion of implant rupture.

\section{MRI}

The MRI results showed signs of rupture in 17 of 50 patients (34\%) and in 22 of 85 implants (26\%). Ten of the 17 patients (59\%) with presumed implant rupture did not show any clinical symptoms, while seven (41\%) showed clinical signs of implant rupture. In 22 implants with signs of rupture, $12(55 \%)$ were symptomatic and ten $(45 \%)$ were asymptomatic (Figure 3A). Of these 22 implants, 19 were diagnosed with the linguine sign and three with the keyhole sign on MRI as evidence for implant rupture (Figure 3B). Eight of 19 (42\%) implants with the linguine sign and two of three (67\%) implants showing the keyhole sign had been asymptomatic.

A

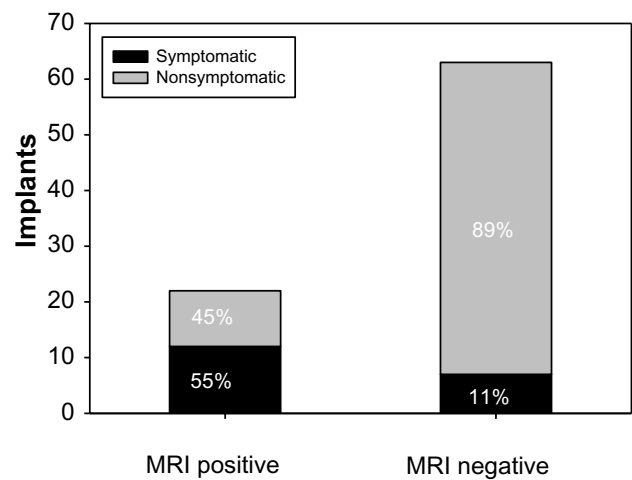

\section{Intraoperative diagnosis}

Twelve of the 17 patients with a positive MRI examination underwent surgical removal of their implants. The remaining five patients were all asymptomatic and refused to undergo surgery. Altogether, 17 implants were removed. The suspected rupture was confirmed intraoperatively in seven of the $17(41 \%)$ patients with explanted breast implants. Of these seven patients, four $(57 \%)$ had been symptomatic. In ten of the 17 patients (59\%), no implant rupture was identified during the operation. Of these ten patients, nine $(90 \%)$ had been symptomatic beforehand (Figure 4). On ultrasound imaging of the harvested implants, we found signs of interruption of the inner layers of the implant shell despite integrity of the outer shell (Figure 5A-C).

\section{Experimental simulation of implant rupture signs}

Figure $2 \mathrm{C}$ shows the MRI of an unused intact silicone gelfilled breast implant, which has been compressed during MRI examination. The simulated complex radial folds are difficult to differentiate from the keyhole sign. As seen in the ultrasound imaging of a new nonruptured implant (Figure 5A),

\section{B}

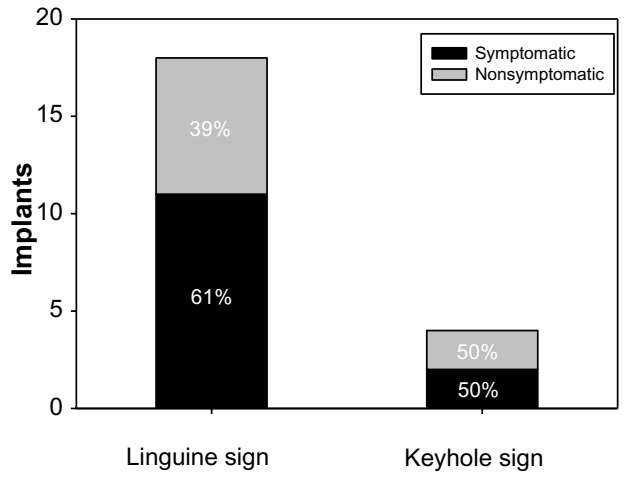

Figure 3 Results of MRI screening for 50 consecutive patients.

Notes: (A) Twenty-two of 85 implants showed signs of rupture, while 63 did not. In patients with positive MRI, 55\% were symptomatic, while II\% in patients with negative MRI were symptomatic. (B) On MRI, most implants were judged to show the linguine sign, and of those, $61 \%$ were symptomatic.

Abbreviation: MRI, magnetic resonance imaging. 


\section{7 implants removed} (positive MRI)

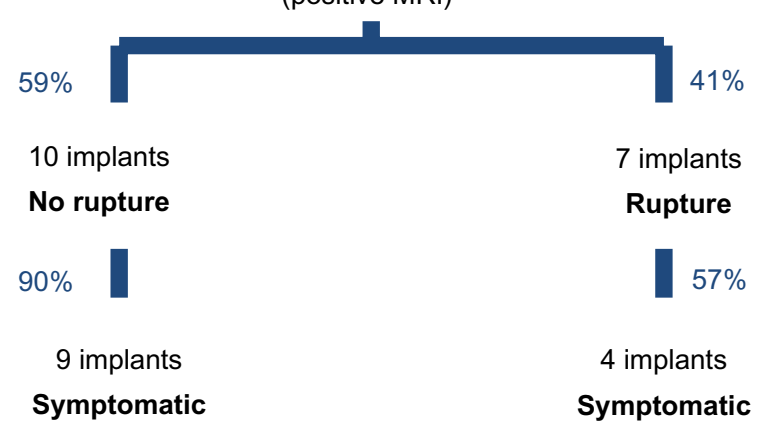

Figure 4 Intraoperative findings after removal of 17 of 22 implants with a positive magnetic resonance imaging result.

the shell consists of three different layers. In implants with a positive linguine sign on MRI, we found alterations in the shell structure with silicone gel in between the layers (Figure 5B). In one case, we were able to find the broken inner layer floating in the silicone gel (Figure 5C).

An intact unused silicone-gel filled breast implant was dissected by a clean cut of the shell in order to experimentally simulate the linguine sign. The different layers of the implant were then dissected, after which the shell was closed again with sutures (Figure 6A and B). MRI of the microsurgically prepared implant showed a low signal intensity line in the high signal intensity silicone gel, mimicking the linguine sign (Figure 6C).

\section{Discussion}

Ever since the introduction of silicone gel breast implants in the early 1960s, plastic surgeons have been faced with the short-term and long-term consequences. Among the various sequelae of breast implant placement, capsular contracture, asymmetry, and implant malposition are the most frequent. ${ }^{10,11}$ However, the possibility of implant rupture and potential leakage of silicone into the body has been of ongoing concern. In 2010, of the nearly 300,000 breast augmentations and 93,000 breast reconstructions performed in the USA, 51\% and 59\%, respectively, were performed with silicone implants. ${ }^{11}$ It can be assumed that these numbers are considerably higher in other western countries, in which no temporary ban on the use of silicone implants was in effect. Rupture of silicone implants was reported in $8 \%$ of asymptomatic women ${ }^{12}$ and in about $33 \%$ of symptomatic women. ${ }^{13-15}$ Symptoms are generally defined as the presence of new onset of pain, capsular contracture, shape change of the implant, and breast asymmetry. Implant rupture in asymptomatic patients is usually suspected during imaging as part of routine screening. ${ }^{2}$ The risk for implant rupture increases with the age of the implant. A rupture prevalence of $30 \%$ at an implant age of 5 years, $50 \%$ at 10 years, and $70 \%$ at 17 years has been reported. ${ }^{15}$ The median age of implant at rupture has been estimated to be 10.8 years. ${ }^{12,16}$

The clinical diagnosis of implant rupture is difficult, considering the manifoldness of potential clinical rupture signs. The sensitivity of physical examination of the breast for detection of implant rupture was shown to be as low as $30 \% .{ }^{17}$ Therefore, MRI has evolved over the last 10 years to become the number one imaging tool for identification of both intracapsular and extracapsular implant rupture. Mammography and computed tomography have been abandoned due to specific drawbacks, including the risk of implant damage and radiation exposure. ${ }^{2}$ Ultrasound imaging may be the way of the future. However, ultrasound imaging is highly operator-dependent and detection of intracapsular rupture relies largely on the experience of the examiner. ${ }^{18}$

In light of this, it was the aim of this study to investigate the ability of MRI screening to accurately detect implant rupture in 50 consecutive patients. However, due to the fact that only 12 of 17 symptomatic women underwent implant removal, and not all women were examined by MRI, statistical variables like sensitivity and specificity were not defined. Explantation of all implants, ie, including those in asymptomatic women with a negative MRI screen, would not have been clinically feasible. We found a strikingly low correlation
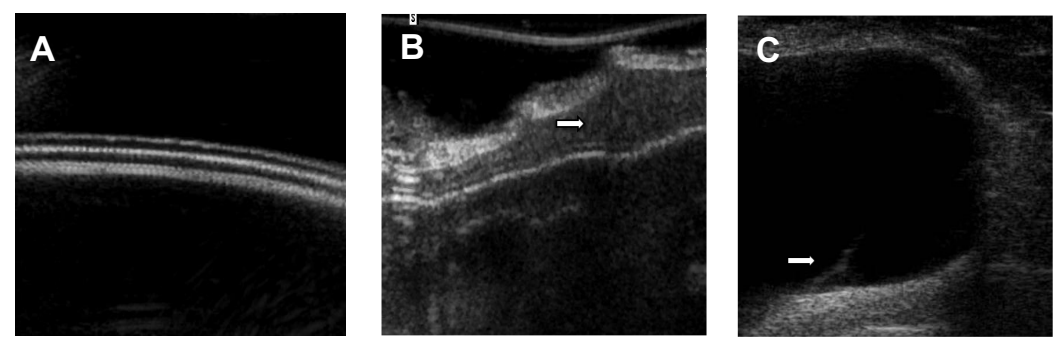

Figure 5 Ultrasound imaging.

Notes: (A) Three separate shell layers can be identified in an intact silicone gel breast implant. (B) Silicone gel found in between the dissolving shell layers of a harvested implant with positive linguine sign on magnetic resonance imaging (arrow). (C) Broken inner shell layer floating in the intraluminal silicone gel of an implant with the linguine sign on magnetic resonance imaging (arrow). 

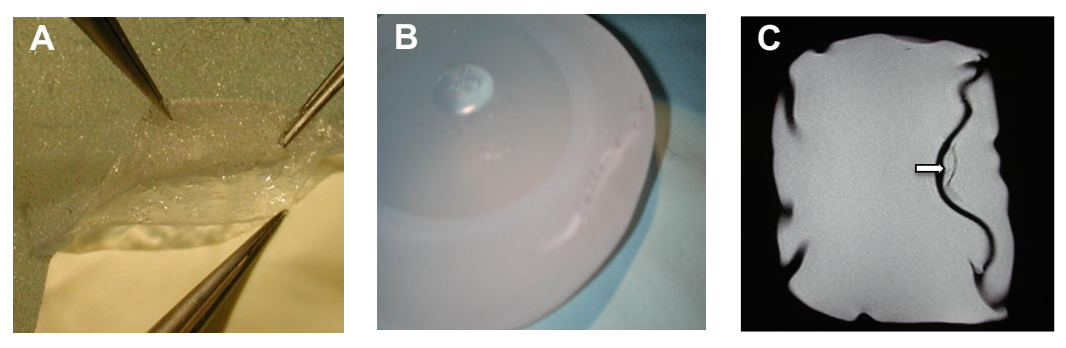

Figure 6 (A) Microsurgical separation of the different shell layers and (B) suture of the outer layer. (C) Low signal intensity line in the high signal intensity silicone gel seen on magnetic resonance imaging after microsurgical separation of the shell layers, mimicking the linguine sign (arrow).

between supposed implant rupture on MRI examination and the intraoperative implant condition. A positive MRI finding was congruent with implant rupture in only $41 \%$ of cases, while the MRI result was false positive for $59 \%$ of implants. Moreover, of the 59\% intact implants mentioned above, $90 \%$ had been symptomatic. This clearly demonstrates that there was no coherence between clinical symptoms and actual presence of implant rupture in the study population.

However, MRI is still assumed to be the best imaging modality available for the diagnosis of implant rupture, with a reported sensitivity and specificity of $>90 \%{ }^{5,9,17,19}$ Implant rupture is basically divided into two categories, ie, extracapsular rupture, referring to free silicone outside the fibrous capsule formed by the body around the implant, and intracapsular rupture, in which the silicone gel is still contained within the fibrous capsule. ${ }^{9}$ Eighty to $90 \%$ of ruptures are intracapsular. ${ }^{5,20}$ The most recent, so-called third-generation, silicone gel implants, which were introduced in the late $1980 \mathrm{~s}$, consist of a cohesive viscous silicone gel encapsulated in a silicone shell. The older secondgeneration silicone gel breast implants that were implanted before the late 1980s consist of a shell filled with less viscous silicone gel.

Based on the fact that the composition of the implant core is different, each implant generation has specific MRI findings for detection of an implant rupture. The secondgeneration of silicone gel implants tends to give the image of the classic collapsed implant shell floating in the more liquid silicone core, creating the linguine sign. In contrast, the third generation of silicone gel implants consisting of a more cohesive silicone gel typically shows an uncollapsed rupture, creating the keyhole sign. Therefore, third-generation implants will rarely have the totally collapsed implant shell creating the linguine sign. ${ }^{9,21}$

In the present study, the patients had had the implant in place for an average of 3.8 (0.2-28) years. This means that the large majority of patients had implants belonging to the third generation that were implanted in the 1990s, even though a small number may have carried second-generation implants. Overall, in 18 implants, the linguine sign was diagnosed on MRI and in only two implants the keyhole sign was found. Based on the abovementioned assumptions, it is highly likely that most changes in the inner structure of the breast implant that were interpreted as linguine signs indicating the silicone shell floating in the liquid gel were actually artifacts caused by tears in the more cohesive gel of third-generation implants. The implant shell itself in these cases was intact. On ultrasound, we were able to show tears in the viscous silicone gel. In an attempt to mimic possible artifacts during breast MRI, we compressed an intact silicone implant, yielding deep folds on MRI, which could be misinterpreted as keyhole signs. Also, microdissection of the implant shell from the cohesive silicon core in a third-generation implant leads to the appearance of curved lines similar to the linguine sign seen on MRI. Therefore, many linguine signs diagnosed on MRI may be merely tears within the highly cohesive gel of third-generation implants without rupture of the implant shell. These findings should be taken into consideration by radiologists involved in breast implant rupture diagnostic today. The linguine sign is still often judged as a typical sign of intracapsular rupture, ignoring the fact that most implants likely belong to the third generation, and will rarely show this particular sign of rupture.

Microscopic silicone leakage through an intact implant shell is referred to as a gel bleed. ${ }^{5,22}$ The majority of gel bleeds cannot be detected by MRI. If a larger amount of gel bleed collects in a radial fold of an implant, the keyhole sign can develop. In a study reported by Chung et al in asymptomatic women with a low prevalence of rupture (8\%), the predictive value of a positive test was low for both ultrasound (19\%) and MRI (20\%). On the other hand, in symptomatic women, with a rupture prevalence of $33 \%$, the predictive value of a positive test was higher for both ultrasound (68\%) and MRI $(81 \%){ }^{2}$ This means that these modalities are better for detecting implant ruptures in a study population with a high incidence and do not perform well as pure screening modalities. 
Therefore, in the general population of women having silicone breast implants and in which the prevalence of implant rupture is significantly lower, the positive predictive value of MRI is too low to make it suitable as a screening method. ${ }^{23}$ This again is supported by the results of our study. MRI was able to accurately identify implant rupture in 55\% of symptomatic implants, but was able to do this in the asymptomatic group in only $11 \%$ of cases.

Unnecessary explantation of a breast implant can result in considerable esthetic impairment and potential perioperative and anesthesia-related complications. Moreover, it is to be avoided from an economic point of view. The national average for surgeon/physician fees in 2010 for breast augmentation was $\$ 3,351$ and for removal was $\$ 2,288 .{ }^{11}$ After the US Food and Drug Administration re-permitted the use of silicone gel-filled breast implants in 2006, they recommended yearly MRI screening in all women carrying silicone breast implants to rule out asymptomatic ruptures. ${ }^{23}$ However, the consequences of silent silicone implant rupture were shown to be minimal in a prospective study of 271 women with cosmetic breast implants. ${ }^{24}$ In that study, the majority of the women with implant rupture had no visible MRI changes in their ruptured implants over a period of 2 years. In $11 \%$ of implants, the authors observed progression of silicone migration, either as a conversion from intracapsular into extracapsular rupture, as progression of extracapsular silicone, or as increasing herniation of the silicone within the fibrous capsule. However, in most cases, these changes were minor. In the same study, no increase in autoantibody levels was seen during the study period in either study group. From their results, the authors concluded that rupture is a "relatively harmless condition, which only rarely progresses and gives rise to notable symptoms". ${ }^{24}$ Song et al studied the diagnostic accuracy of MRI examination regarding breast implant rupture, and found that most studies using MRI and ultrasound examined symptomatic subjects and therefore had a 14-fold higher diagnostic accuracy than studies in asymptomatic subjects. ${ }^{25}$ This underlines the lack of proof that routine screening of patients with breast implants will result in correct detection of implant ruptures. Likewise, McCarthy et al stress this aspect in their evaluation of the ability of MRI screening to provide health benefits for women with breast implants. ${ }^{26}$

The consequences of the two types of implant rupture have to be considered when making decisions. In asymptomatic patients with an implant rupture, the integrity of the implant shell becomes impaired yet no symptoms are experienced by the patient and there are no obvious changes in the shape of the breast or implant. In contrast, in patients with a symptomatic rupture, silicone gel leaks from the implant shell into the intracapsular and/or extracapsular space, which results in a change in breast appearance and/or development of silicone granulomas or axillary lymphadenopathy. ${ }^{26-28}$

In conclusion, sole reliance on MRI findings in asymptomatic patients will most likely result in a larger number of unnecessarily explanted implants. On the other hand, pure clinical examination will be limited in its ability to accurately and definitively confirm or rule out a potential implant rupture. Therefore, the decision to reoperate has to be made carefully and in agreement with the patient, after all advantages and disadvantages as well as risks of such a procedure have been weighed up. The tolerance level of each individual patient for insecurity regarding a potentially silent ruptured silicone implant within the body should also be taken into account. If the patient decides against explantation, close clinical and radiological examination of the breast should be performed to detect possible progression of a silicone leak.

\section{Acknowledgment}

Study was supported in part by the Medical-Scientific Fund of the Mayor of Vienna, Project \# 2107.

\section{Disclosure}

The authors report no conflicts of interest in this work.

\section{References}

1. US Food and Drug Administration. FDA approves silicone gel-filled breast implants after in-depth evaluation. Available from: http://www. fda.gov/newsevents/newsroom/pressannouncements/2006/ucm108790. $\mathrm{htm}$. Accessed November 2, 2011.

2. Chung KC, Malay S, Shauver MJ, Kim HM. Economic analysis of screening strategies for rupture of silicone gel breast implants. Plast Reconstr Surg. 2012;130:225-237.

3. Press RI, Peebles CL, Kumagai Y, Ochs RL, Tan EM. Antinuclear autoantibodies in women with silicone breast implants. Lancet. 1992;340:1304-1307.

4. Holmich LR, Lipworth L, McLaughlin JK, Friis S. Breast implant rupture and connective tissue disease: a review of the literature. Plast Reconstr Surg. 2007;120(7 Suppl 1):62S-69S.

5. Gorczyca DP. MR imaging of breast implants. Magn Reson Imaging Clin NAm. 1994;2:659-672.

6. Ikeda DM, Borofsky HB, Herfkens RJ, Sawyer-Glover AM, Birdwell RL, Glover GH. Silicone breast implant rupture: pitfalls of magnetic resonance imaging and relative efficacies of magnetic resonance, mammography, and ultrasound. Plast Reconstr Surg. 1999;104: 2054-2062.

7. Caskey CI, Berg WA, Hamper UM, Sheth S, Chang BW, Anderson ND. Imaging spectrum of extracapsular silicone: correlation of US, MR imaging, mammographic, and histopathologic findings. Radiographics. 1999;19 Spec No:S39-S51.

8. Cher DJ, Conwell JA, Mandel JS. MRI for detecting silicone breast implant rupture: meta-analysis and implications. Ann Plast Surg. 2001;47:367-380. 
9. Gorczyca DP, Gorczyca SM, Gorczyca KL. The diagnosis of silicone breast implant rupture. Plast Reconstr Surg. 2007;120(7 Suppl 1): 49S-61S.

10. Hvilsom GB, Holmich LR, Henriksen TF, Lipworth L, McLaughlin JK, Friis S. Local complications after cosmetic breast augmentation: results from the Danish Registry for Plastic Surgery of the Breast. Plast Surg Nurs. 2010;30:172-179.

11. American Society of Plastic Surgeons. National clearinghouse of plastic surgery procedural statistics. Available from: http://www.plasticsurgery. org/news-and-resources/statistics. html. Accessed October 20, 2011.

12. Heden P, Nava MB, van Tetering JP, et al. Prevalence of rupture in inamed silicone breast implants. Plast Reconstr Surg. 2006;118:303-308.

13. Goodman CM, Cohen V, Thornby J, Netscher D. The life span of silicone gel breast implants and a comparison of mammography, ultrasonography, and magnetic resonance imaging in detecting implant rupture: a meta-analysis. Ann Plast Surg. 1998;41:577-585.

14. Kessler DA. The basis of the FDA's decision on breast implants. N Engl J Med. 1992;326:1713-1715.

15. Netscher DT, Weizer G, Malone RS, Walker LE, Thornby J, Patten BM. Diagnostic value of clinical examination and various imaging techniques for breast implant rupture as determined in 81 patients having implant removal. South Med J. 1996;89:397-404.

16. Marotta JS, Widenhouse CW, Habal MB, Goldberg EP. Silicone gel breast implant failure and frequency of additional surgeries: analysis of 35 studies reporting examination of more than 8,000 explants. J Biomed Mater Res. 1999;48:354-364.

17. Holmich LR, Fryzek JP, Kjøller K, et al. The diagnosis of silicone breast-implant rupture: clinical findings compared with findings at magnetic resonance imaging. Ann Plast Surg. 2005;54:583-589.

18. Azavedo E, Bone B. Imaging breasts with silicone implants. Eur Radiol. 1999;9:349-355.

19. Everson LI, Parantainen H, Detlie T, et al. Diagnosis of breast implant rupture: imaging findings and relative efficacies of imaging techniques. AJR Am J Roentgenol. 1994;163:57-60.
20. Gorczyca DP, DeBruhl ND, Mund DF, Bassett LW. Linguine sign at MR imaging: does it represent the collapsed silicone implant shell? Radiology. 1994;191:576-577.

21. Collis N, Sharpe DT. Silicone gel-filled breast implant integrity: a retrospective review of 478 consecutively explanted implants. Plast Reconstr Surg. 2000;105:1979-1985.

22. Middleton MS, McNamara MP, editors. Breast Implant Imaging. Philadelphia, PA, USA: Lippincott Williams \& Wilkins; 2003.

23. Anaplastic large cell lymphoma (ALCL) in women with breast implants: preliminary FDA findings and analyses. Center for Devices and Radiological Health, US Food and Drug Administration; 2011. Available from: http://www.fda.gov/medicaldevices/ productsandmedicalprocedures/implantsandprosthetics/breastimplants/ ucm239996.htm. Accessed March 22, 2014.

24. Holmich LR, Vejborg IM, Conrad C, et al. Untreated silicone breast implant rupture. Plast Reconstr Surg. 2004;114:204-214.

25. Song JW, Kim HM, Bellfi LT, Chung KC. The effect of study design biases on the diagnostic accuracy of magnetic resonance imaging for detecting silicone breast implant ruptures: a meta-analysis. Plast Reconstr Surg. 2011;127:1029-1044.

26. McCarthy CM, Pusic AL, Kerrigan CL. Silicone breast implants and magnetic resonance imaging screening for rupture: do US Food and Drug Administration recommendations reflect an evidence-based practice approach to patient care? Plast Reconstr Surg. 2008;121: 1127-1134.

27. Teuber SS, Reilly DA, Howell L, Oide C, Gershwin ME. Severe migratory granulomatous reactions to silicone gel in 3 patients. J Rheumatol. 1999;26:699-704.

28. Brown SL, Pennello G, Berg WA, Soo MS, Middleton MS. Silicone gel breast implant rupture, extracapsular silicone, and health status in a population of women. J Rheumatol. 2001;28:996-1003.
International Journal of Women's Health

\section{Publish your work in this journal}

The International Journal of Women's Health is an international, peerreviewed open-access journal publishing original research, reports, editorials, reviews and commentaries on all aspects of women's healthcare including gynecology, obstetrics, and breast cancer. The manuscript management system is completely online and includes

\section{Dovepress}

a very quick and fair peer-review system, which is all easy to use. Visit http://www.dovepress.com/testimonials.php to read real quotes from published authors. 